\title{
ANÁlisis DEL NIVEL dE ATENCIÓN DE LOS ALUMNOS EN CLASE UTILIZANDO NEUROSKY'S MINDWAVE MOBILE
}

ANALISYS OF STUDENTS' ATTENTION LEVEL IN CLASSROOM USING NEUROSKY'S MINDWAVE MOBILE

Cristóbal Romero Morales, Jose María Luna Ariza, Alejandro Bogarín Vega, María Luque Rodríguez, Pedro Gonzalez Espejo cromero@uco.es

Received: 28/06/2017 Accepted: 20/02/2018

\begin{abstract}
Resumen
Este artículo describe un software en Android específico para el dispositivo NeuroSky's MindWave Mobile, con el que poder medir el nivel de atención de los estudiantes de grado en informática durante las sesiones tradicionales de clases teóricas y prácticas. Utilizando este software se podrá monitorizar a un grupo de alumnos que portarán el dispositivo en sus cabezas como si fueran unos típico auriculares de escuchar música. El software específico desarrollado irá recogiendo en tiempo real la información sobre el nivel de atención directamente desde el propio teléfono móvil de alumno. A partir de esta información los profesores pueden analizar y determinar: en que sesiones los alumnos han mostrado más o menos atención, en que momentos de cada clase muestran más o menos atención, que transparencias o contenidos son en las que muestran más o menor atención, que comentarios producen mayor atención o menor atención en clase, que alumnos muestran más atención en teoría o en práctica, etc. y por tanto se podrán determinar multitud de posibles acciones para intentar aumentar el nivel de atención de los alumnos durante sus clases.
\end{abstract}

Palabras clave: Nivel de Atención; Onda Celebral, Aplicación Android.

\section{Abstract}

This paper describes a specific Android software to the NeuroSky's MindWave Mobile device in order to measure the students' attention level during the traditional theory and practical classroom sessions. Using this software we can monitor to a group of students carried out the device in their heads as a typical headphones. This software will gather on real time all the information about the attention level directly on the smartphone of each student. Starting from this information, instructors can analyse and determine in which sessions the students has shown more or less attention, in which slides or contents has shown more or less attention, which comments in classroom generate a higher attention, which students show a higher attention in theory or practical sessions, etc. and so, they can determine some possible actions in order to try to increase the attention level in classroom.

Keywords: Attention Level; Brain Wave; Android Application.

\section{INTRODUCCIÓN}

El nivel de atención es uno de los factores que afecta en el buen rendimiento académico de los estudiantes. Hoy sabemos que la atención no constituye un proceso cerebral único sino que existen diferentes redes atencionales que hacen intervenir circuitos neuronales y regiones cerebrales concretas (Posner y otros, 2007). Aunque existen también mecanismos inconscientes que permiten mantener la atención, nosotros nos centraremos en los aspectos voluntarios y conscientes de la atención que conlleva lo que conocemos como concentración y que desempeña un papel esencial en una forma de memoria (memoria explícita) que es la forma de aprendizaje utilizada cuando por ejemplo los alumnos reciben una clase de teoría o prácticas. Diversos estudios demuestran que la capacidad del alumno para mantener la atención sostenida varía, en promedio, entre 10 y 20 minutos (Tokuhama, 2011). El intentar mantener la atención durante periodos de tiempo prolongados agota determinados neurotransmisores de la corteza prefrontal. Esto sugiere por ejemplo que, para optimizar la atención del alumnado, el profesor podría dividir el tiempo que dispone para impartir su materia en bloques que no excedieran, aproximadamente, los 15 minutos. De esta forma también se facilita el procesamiento y consolidación de la información que sabemos que requiere práctica continua y tiempo. También se puede tener en cuenta que en el inicio de la clase se debería despertar el interés, en la mitad de la misma se podría facilitar la reflexión a través del trabajo cooperativo y utilizar el final para repasar lo prioritario. Existe una gran diversidad de estrategias pedagógicas que pueden estimular al cerebro y captar la atención siempre y cuando conlleven cambio y novedad. Desde la utilización por parte del docente, por ejemplo, de metáforas, historias, ejercicios que propongan predicciones, actividades que requieran analizar diferencias (Jensen y otros, 2013), debates, lecturas o videos hasta cambios regulares en el entorno físico de aprendizaje que constituye el aula y que suministren estimulación visual. La experiencia del profesor permitirá mantener ese equilibrio requerido entre lo novedoso y lo más tradicional para no provocar estrés inadecuado en el alumnado. Finalmente, tanto la forma o estilo de enseñar del profesor como la utilización de diferentes materiales multimedia pueden ayudar a mantener la curiosidad o el interés del alumno durante una sesión de clase y hacerla mucho más atractiva y menos pesada para el alumno. 
Por otro lado, ya se han comenzado a realizar estudios sobre la monitorización de ondas cerebrales para detectar a través de los denominados BCI (Brain Computer Interfaces) capacidades cognitivas como la concentración o nivel de atención de los alumnos durante las clases (Sezer y otros, 2015). Los dispositivos que permiten capturar esta información son los electroencefalogramas (EEG), con los que es posible monitorear las ondas electromagnéticas generadas por el cerebro para poder ser interpretadas según el comportamiento cerebral del usuario. En sus inicios la información captada por los dispositivos EEG, fue normalmente utilizada con fines de estudios médicos, apoyando a la salud mental de las personas. Estos primeros dispositivos eran muy caros y aparatosos. Pero conforme la tecnología ha ido avanzando, se han ido reduciendo en precio, se han ido haciendo portables, y aumentando su conectividad hasta pasar al mercado de consumo masivo. Por lo que en la actualidad, estos dispositivos proporcionan de un preciso instrumento de medición de la frecuencia de nuestras ondas cerebrales para monitorizarla y mostrarla normalmente en la pantalla de un ordenador. Actualmente, existen distintos dispositivos BCI comerciales, pero entre todos ellos cabe destacar el Neurosky's MindWave Mobile (https://www.mindtecstore.com/en/mindwave-mobile-brainwave-starter-kit), ver Figura 1.

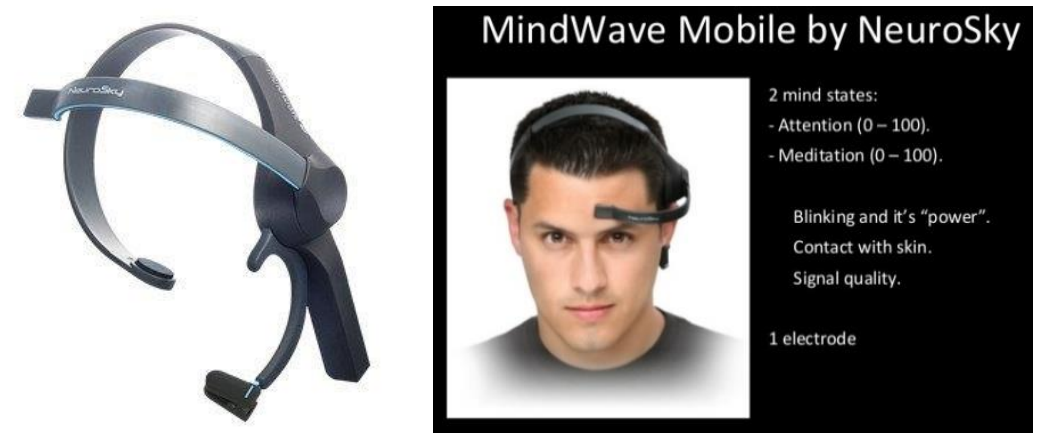

Figura 1. Neurosky's MindWave Mobile device.

tilizando sólo tres electrodos (debijo de las orejas y la frent integrados algoritmos Theta, Alfa, Beta, y Gamma Por los anteriores motivos, en este proyecto se va proponer realizar un análisis del nivel de atención de los alumnos en clase utilizando dispositivos NeuroSky's MindWave Mobile que llevarán alumnos puestos durante las sesiones de teoría y/o prácticas para monitorizar su nivel de interés.

2. OBJETIVOS

Nuestro principal objetivo es el de poder realizar una mejora continua de las clases presenciales tradicionales de teoría y prácticas, concretamente detectando cuando hay falta de atención o concentración de los estudiantes de grado de la Universidad de Córdoba. Para ello proponemos el uso de los dispositivos BCI y lectura EEG (concretamente el NeuroSky's MindWave Mobile), con el fin de poder obtener información de tipo contextual en tiempo real sobre el estado cognitivo (nivel de atención) en el que se encuentran los estudiantes durante las sesiones de clases presenciales. Además se grabará la clase en video desde el punto de vista del profesor, es decir, con la propia cámara del ordenador portátil del profesor y apuntando hacia profesor. De esta forma se grabará todo lo que hace y dice el profesor durante la clase, las transparencias que utiliza en cada momento, lo que escribe en la pizarra, etc.

Para alcanzar este objetivo principal, también nos planteamos un segundo objetivo que es el de desarrollar un software propio específico para capturar la información del dispositivo NeuroSky directamente desde el móvil del estudiante y poder enviarlo al profesor. De esta forma, el propio estudiante capturará sus datos de nivel de atención desde su propio teléfono móvil y será el encargado en enviárselos al profesor. Nos hemos planteado este objetivo, debido a que la empresa NeuroSky sólo proporciona gratuitamente software de visualización de datos, que no permite la captura, almacenamiento y análisis de los datos, sino simplemente la visualización en un PC (http://store.neurosky.com/products/visualizer-2-0). Sin, embargo, la empresa NeuroSky si propociona herramientas y APIs de programación para Windows, Mac, IOS o Android (http://store.neurosky.com/collections/developer-tools-3). Utilizando estas herramientas de desarrollo nos proponemos desarrollar una aplicación específica para dispositivos Android (ya la mayoría de los alumnos tienen Android en sus móviles) que sea capaz de conectarse y gestionar el dispositovo NeuroSky Mindwave Mobile Wireless Headset, mediante tecnología Bluetooth y almacene los datos del nivel de interés en cada instante en un fichero de tipo CSV o Excel. Dichos ficheros se podrán almacenar en la nube, por ejemplo en Dropbox, para posteriormente poderse enviar o compartir con el profesor. Las principales características de la aplicación serán:

- Permitirá que el usuario se identifique con su nombre al entrar a la aplicación.

- Permitirá conectarse fácilmente mediante bluetooth al dispositivo NeuroSky, previamente sincronizado.

- Permitirá iniciar una capturar del nivel de atención en cualquier momento, y parar dicha captura.

- Permitirá visualizar de manera numérica y gráfica los datos que se están recogiendo en tiempo real.

- Permitirá configurar una cuenta de dropbox en la cual se subirán los datos recogidos. 


\section{METODOLOGÍA}

La metodología utilizada para llevar a cabo este proyecto, consta de las dos siguientes actividades principales:

1. Desarrollo de un software específico para la captura de la información del dispositivo NeuroSky directamente desde un móvil. Para ello un alumno de proyecto fin de grado lo ha programado utilizando el lenguaje Android y el API proporcionado por el fabricante.

2. Probar el software para poder recoger datos durante las sesiones de clase. Para ello el profesor podrá grabar en video sus clases desde su propio ordenador, y algunos alumnos elegidos aleatoriamente grabarán su nivel de atención utilizando los dispositivos NeuroSky.

\section{DESCRIPCIÓN}

A continuación se describe la funcionalidad del software Android que hemos denominado EEG Analizer que se ha desarrollado para capturar la información sobre el nivel de atención. Tras descargar e instalar la aplicación en nuestro móvil, tenemos que vincular el dispositivo NeuroSky con nuestro Smartphone vía Bluetooth.

Encenderemos nuestro dispositivo Neurosky y presionaremos el interruptor de encendido de la misma forma que antes hasta que el led azul parpadee dos veces por segundo. Una vez hecho esto, pulsamos el botón con el símbolo del Bluetooth en pantalla principal de la aplicación móvil y la aplicación comenzará a conectarse y nos aparecerá el menú principal (Ver Figura 2, imágen de la izquierda). 

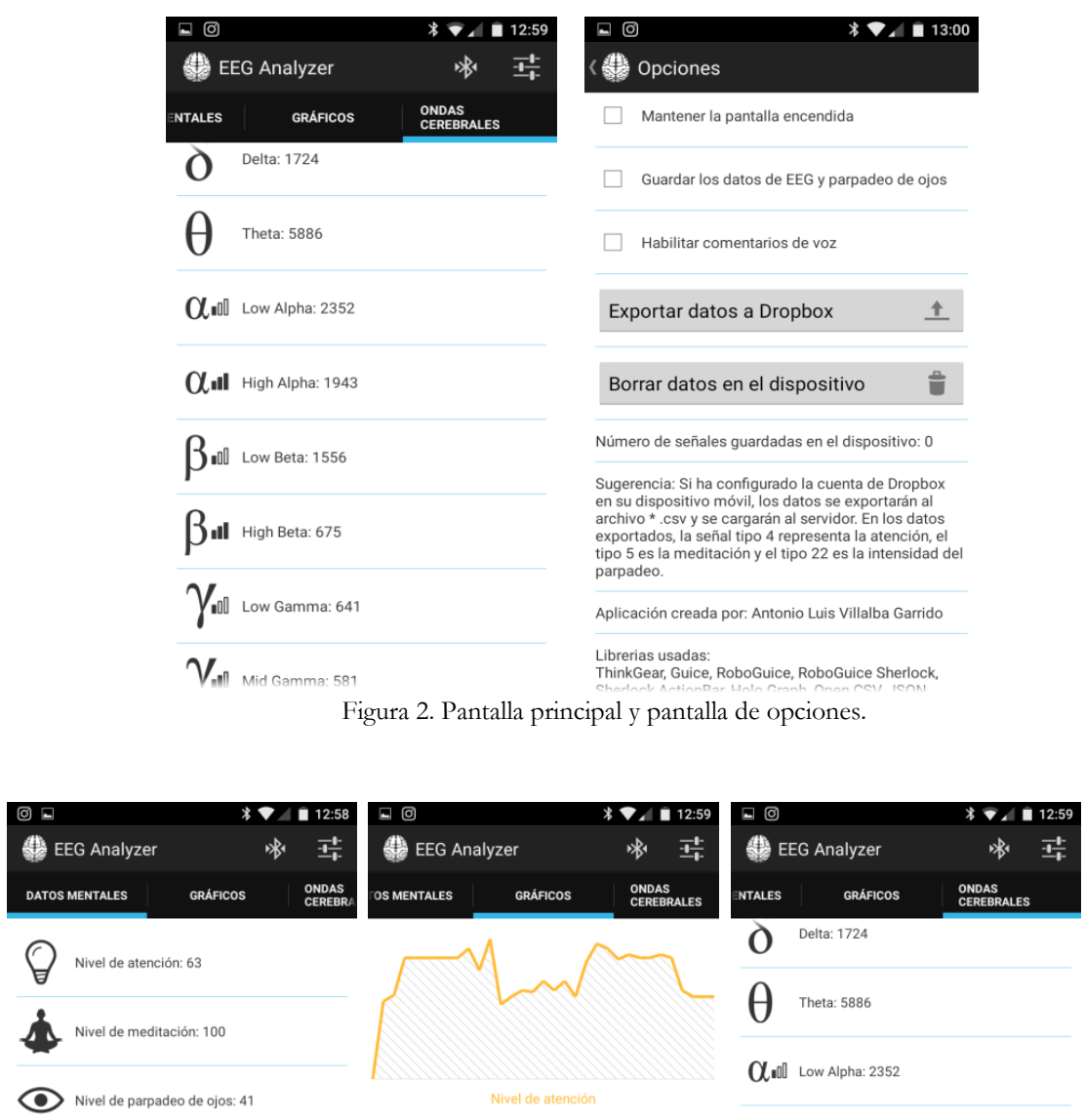

O Deta: 1724

$\theta$ Theta: 5886

Q 100 Low Alpha: 2352

.11ll Datosentroto: :202

Q.ll High Alpha: 1943

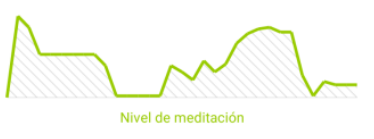

$\beta$ will Low Beta 1556

$\beta$ ul High Beta: 675

$\gamma_{\text {NIl Low Gamma: 641 }}$

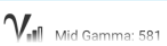

Figura 3. Pantalla de visualización de datos.

En él encontraremos, siguiendo un orden descendente de la pantalla y de izquierda a derecha, un botón para activar o desactivar la conexión con el dispositivo NeuroSky con el símbolo del Bluetooth, un botón que nos llevará al menú de opciones de la aplicación (Ver Figura 2, imágen de la derecha), y en la zona inferior restante las distintas visualizaciones de datos (Ver Figura 3).

En la ventana de visualizacion de datos (Ver Figura 3), podemos ver los datos mentales (nivel de atención, nivel de meditación, nivel de parpadeo y número de datos capturados), los gráficos del nivel de atención y meditación y los valores de las ondas cerebrales (delta, theta, low alpha, high alpha, low beta, high beta, low gamma and mid gamma).

Finalmente, también están las opciones de la aplicación, que se acceden desde el icono superior derecho de la pantalla (Ver Figura 2, imágen de la derecha). Desde las opciones, se puede configurar para mantener la ventana de captura encendida, guardar los datos y habilitar comentarios de voz, además de exportar los datos a Dropbox y borrar los datos del dispositivo.

Si conviene señalar que los datos guardados al señalar la opción correspondiente se almacenan en un fichero .csv, el cual se puede abrir mediante cualquier software que lea hojas excel. 
Cuando decidimos exportar los daos a Dropbox es necesario iniciar sesión con la misma, y una vez hecho esto solo tendremos que pulsar en el botón de la pantalla con el símbolo de Dropbox y se subirá el fichero .csv mencionado anteriormente a dicha cuenta.

\section{RESULTADOS OBTENIDOS}

En la actualidad se están haciendo pruebas de capturas de datos en clases reales con los alumnos de la asignatura de Sistemas Interactivos del Grado en Ingeniería Informática. A continuación se muestra parte de un fichero CSV capturado con un alumno en una clase durante unos segundos (ver Figura 4).

\begin{tabular}{|c|c|c|c|c|c|c|c|c|c|c|}
\hline в & c & D & E & $\mathrm{F}$ & G & H & 1 & 1 & $\mathrm{k}$ & L \\
\hline Attention I & Meditation & Delta & Theta & LowAlpha & HighAlpha L & LowBeta & HighBeta & LowGamma & MiddleGamma & Date \\
\hline 66 & 63 & 440360 & 24228 & 3311 & 16054 & 67838 & 12002 & 3963 & 2439 & Thu Apr 06 10:56:57 GMT+02:00 2017 \\
\hline 54 & 60 & 218448 & 75310 & 46733 & 25638 & 59821 & 10849 & 5030 & 4266 & 5 Thu Apr 06 10:56:58 GMT+02:00 2017 \\
\hline 54 & 48 & 161210 & 33025 & 15167 & 4952 & 4000 & 155 & 1738 & & 7 Thu Apr 06 10:56:59 GMT+02:00 2017 \\
\hline 54 & 50 & 139882 & 21730 & 8942 & 1559 & 2971 & 3555 & 469 & & 3 Thu Apr 06 10:57:01 GMT+02:00 2017 \\
\hline 43 & 61 & 2027298 & 215503 & 94159 & 51958 & 24531 & 39894 & 9883 & 12769 & Thu Apr 06 10:57:02 GMT+02:00 2017 \\
\hline 44 & 61 & 399581 & 123355 & 36265 & 17424 & 13692 & 14914 & 3255 & & 7 Thu Apr 06 10:57:04 GMT+02:00 2017 \\
\hline 43 & 64 & 448202 & 107720 & 66372 & 24848 & 43261 & 17778 & 9166 & & 7 Thu Apr 06 10:57:05 GMT+02:00 2017 \\
\hline 40 & 40 & 157532 & 161761 & 3607 & 4568 & 15947 & 14417 & 5230 & 1973 & 3 Thu Apr 06 10:57:06 GMT+02:00 2017 \\
\hline 26 & 38 & 1169204 & 18427 & 3125 & 9051 & 3137 & 1472 & 311 & & Thu Apr 06 10:57:07 GMT+02:00 2017 \\
\hline 37 & 37 & 2474862 & 324092 & 63995 & 54023 & 82401 & 83524 & 4163 & 1802 & 2 Thu Apr 06 10:57:08 GMT+02:00 2017 \\
\hline 29 & 26 & 365953 & 101413 & 5142 & 867 & 17192 & 6829 & 1273 & 581 & 1 Thu Apr 06 10:57:10 GMT+02:00 2017 \\
\hline 40 & 47 & 1534794 & 88951 & 16502 & 26337 & 49475 & 24835 & 9368 & 2683 & 3 Thu Apr 06 10:57:11 GMT+02:00 2017 \\
\hline 48 & 41 & - 274741 & 101845 & 28987 & 612 & 7504 & 13717 & 247 & 845 & 5 Thu Apr 06 10:57:20 GMT+02:00 2017 \\
\hline 43 & 48 & 3584277 & 529324 & 59277 & 231653 & 67046 & 101433 & 7053 & 4239 & Thu Apr 06 10:57:25 GMT+02:00 2017 \\
\hline 57 & 63 & 1541639 & 15993 & 25649 & 15290 & 25389 & 10073 & 2748 & 3167 & 7 Thu Apr 06 10:57:28 GMT+02:00 2017 \\
\hline 47 & 64 & 93542 & 11089 & 820 & 5166 & 5077 & 1300 & 670 & & Thu Apr 06 10:57:29 GMT+02:00 2017 \\
\hline 50 & 50 & 1324708 & 1081676 & 24953 & 90086 & 272770 & 161257 & 7820 & 5925 & 5 Thu Apr 06 10:57:30 GMT+02:00 2017 \\
\hline 40 & 51 & 3324877 & 118926 & 51844 & 28826 & 24329 & 11384 & 1190 & 1242 & 2 Thu Apr 06 10:57:31 GMT+02:00 2017 \\
\hline 50 & 43 & 286457 & 26408 & 9030 & 36771 & 69100 & 26047 & 11685 & 4709 & Thu Apr 06 10:57:32 GMT+02:00 2017 \\
\hline 40 & 47 & 1395503 & 573249 & 153654 & 165632 & 50438 & 32740 & 6148 & & 7 Thu Apr 061 \\
\hline
\end{tabular}

Figura 4. Ejemplo de fichero generado por la aplicación.

A partir del anterior fichero CSV se puede obtener fácilmente por ejemplo un gráfica del nivel de atención del mismo alumno durante toda la clase (ver Figura 5).

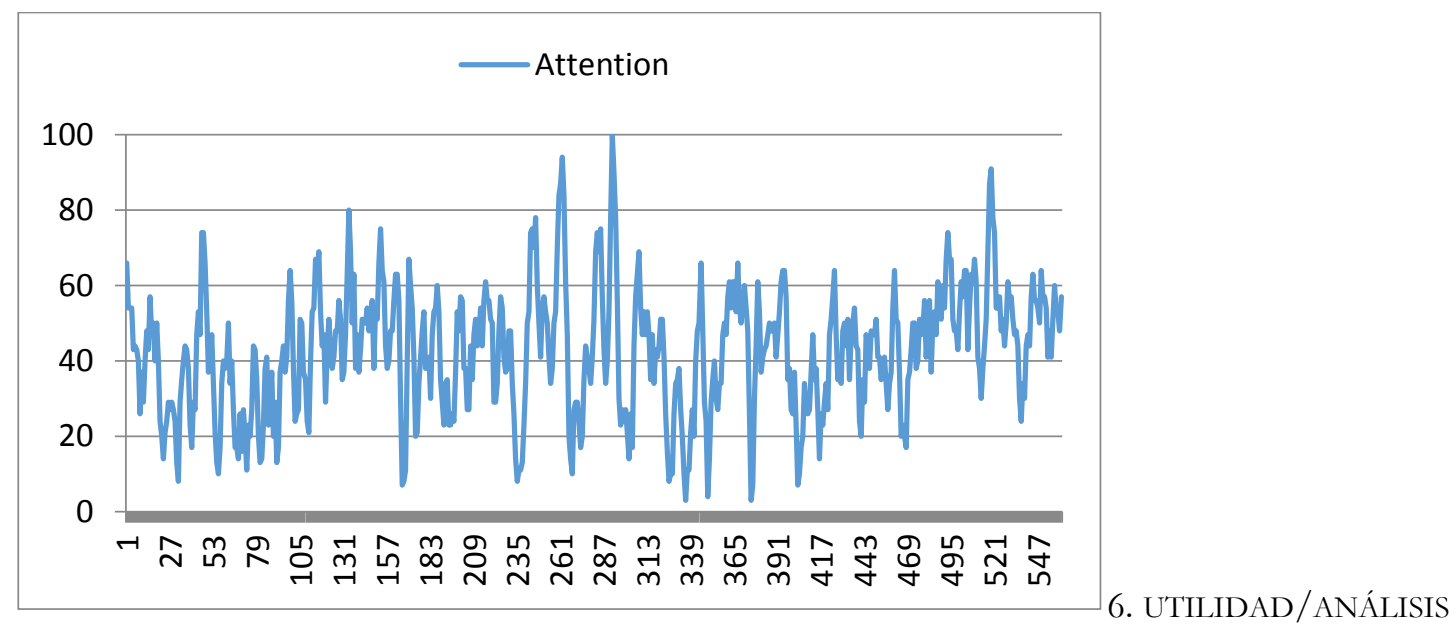

Figura 5. Ejemplo de captura de nivel de atención.

A partir de la información recogida (sobre el interés de los alumnos proporcionado por los dispositivos EEG, que deberá de ser analizada posteriormente por un grupo de profesores (incluido el propio profesor) que intentará descubrir información que permita mejorar el nivel de atención y la disminución de distracciones durante el aprendizaje de los estudiantes en sus clases de teoría y prácticas. Para ello, al conocer que el estado cognitivo se encuentra la mayoría de la clase en un determinado momento no es apto para el aprendizaje, es decir, los estudiantes no están concentrados o se encuentran distraídos, indicará al profesor que puede ocurrir algún tipo de problema, que debería de intentar remediar en sus futuras clases, como podría ser por ejemplo:

- Que el contenido concreto mostrado a los alumnos es demasiado difícil, abstracto, no se entiende, no se lee bien, o bien no se presenta visualmente de la mejor forma para que los alumnos se interesen o muestren alguna curiosidad en él. 
- Que la forma o estilo en la que el profesor presenta el material concreto no es el más adecuado. O bien por cansancio, aburrimiento, etc., puede ocurrir que o bien el nivel de voz, la energía y movimientos del profesor decaigan y no animen al alumno a seguir la explicación.

- Que la duración de las clases se hace demasiado larga, denso y no se le ha dejado ningún momento de relajación, por lo que los alumnos están agotados y no prestan atención.

- Que los estudiantes que han mostrado un nivel más alto de interés, luego son los que obtienen mejores resultados en las actividades de evaluación de la asignatura.

De igual forma el profesor puede determinar en qué clases, sesiones e instantes se producen los picos tanto de máxima atención, para determinar así los posibles motivos de más interés/curiosidad para el alumno, y utilizarlos en otros momentos de las clases, como podría ser:

- Que al enseñar contenidos multimedia, vídeos, imágenes, etc. los niveles de atención suben mucho.

- Que el dejar algunos instantes de relajación entre la explicación de conceptos, o entre un ejercicios y otro, hace que suban después el nivel de atención nuevamente.

- Que al explicar de pie y próximo al alumno, en lugar de sentado en la mesa del profesor, hace que suba la atención.

- Que el subir en determinados momentos el tono o voz para explicar conceptos importantes, hace que suba la atención.

- Que el contar alguna historia real, chiste, anécdota más o menos relacionada con el contenido de la clase, puede hacer que se mantenga la atención.

\section{CONCLUSIONES/DISCUSIÓN}

En este proyecto se ha descrito una aplicación Android para medir el nivel de atención de los estudiantes durante la impartición de clases reales de teoría y prácticas. Los alumnos deberán de portar un dispositivo denominado Neurosky's MindWave Mobile que se conecta mediante Bluetooth con un teléfono Android. La aplicación permite ademas de proporcionar información sobre los niveles de las ondas cerebrales Delta, Theta, Alfa, Beta, y Gamma, medir el nivel de atención de los estudiantes. Durante el proyecto sólo se ha podido utilizar los casos MidWave y el software con algunos alumnos de la asignatura Sistemas Interactivos para realizar pruebas de funcionamiento del software y no para capturar datos reales durante la duración de toda una clase. Por tanto, en una siguiente fase, se recogerán datos reales de varios alumnos durante las clases y se analizará todos los datos recogidos durante la impartición de las clases.

\section{BIBLIOGRAFÍA}

JENSEN, ERIC y SNIDER, CAROL. Turnaround tools for the teenage brain. Jossey-Bass. 2013.

Posner, Michael I. Y ROTHBART, MARY K.. “Educating the human brain”. American Psychological Association. 2007.

SEZER, A. İNEL, Y., SECKIN, A., U. UluCINAR, U. “An Investigation of University Students' Attention Levels in Real Classroom Settings with Neurosky's Mindwave Mobile (EEG) Device”. International Educational Technology ConferenceIECT, Bildiri, 88-101. 2015

TOKUHAMA-ESPINOSA, TRACEY. Mind, brain, and education science. A comprehensive guide to the new brain-based teaching. W. W. Norton \& Company. 2011 